\title{
Pollen-related food allergy in children with seasonal allergic rhinitis
}

\author{
Beata Cudowska, Magdalena Pawłowicz, Dariusz M. Lebensztejn
}

Department of Paediatrics, Gastroenterology, Hepatology, Nutrition and Allergology, Medical University of Bialystok, Bialystok, Poland Adv Dermatol Allergol 2021; XXXVIII (1): 96-101 DOI: https://doi.org/10.5114/ada.2021.104284

\begin{abstract}
Introduction: Seasonal allergic rhinitis (SAR) and bronchial asthma are typical manifestations of pollen-food sensitization in adults. There is limited information regarding the sensitization patterns of pollen-food allergy in children. Aim: To evaluate the prevalence of SAR in children with pollen-food sensitization and assess the impact of food allergens on nasal symptoms.

Material and methods: Forty-three children with pollen-food sensitization aged 2-14 years were evaluated for evidence of SAR. The inclusion criteria was IgE-mediated sensitization to pollen and homologous food allergens. The control group consisted of 19 children with SAR caused by pollen without sensitization to homologous food allergens.

Results: Allergic rhinitis was the main symptom in $65.1 \%$ of children; in $25.6 \%$ an association between ingestion of pollen-related foods and nasal symptoms was observed. The simultaneous sensitization to animal origin food allergens was stated in $63.3 \%$ of children with SAR. In $25.6 \%$ anaphylactic reactions to foods were registered. $37.2 \%$ of children were asymptomatic to pollen origin foods despite pollen-food sensitization. The statistically significant differences between values were noticed in comparison to the control group.

Conclusions: Allergic rhinitis is a common manifestation of pollen-food sensitization in children and this type of immunological hyperreactivity should be taken into account regardless of age. The lack of association of symptoms with plant origin foods in the majority of cases and the asymptomatic course of food sensitization in more than one third of patients indicates the need for follow-up and being careful in routine recommending the avoidance diet.
\end{abstract}

Key words: cross-allergy, food-related symptoms, nasal symptoms, anaphylaxis, childhood.

\section{Introduction}

The results of different clinical studies show that people sensitized to pollen, react to plant-derived, fresh foods that can cause allergic symptoms generally limited to the oral mucosa. The mouth and throat itching, tongue swelling, oropharynx angioedema belong to the best-known symptoms and they occur immediately after the food has been swallowed [1-3]. In patients with reactions limited to the oropharynx, the term "oral allergy syndrome" (OAS) is widely used [3, 4]. Only a small group of affected individuals demonstrate systemic allergic reactions such as nausea, diarrhoea, vomiting, generalized urticaria, shortness of breath or even anaphylactic shock (pollen-food syndrome - PFS) [3, 4]. Considering the immunological aspect, the pollen-food syndrome results from the cross-reactivity between pollen-specific IgE and homologous proteins found in fruits, vegetables and some tree nuts [1-3]. Clinical expression is determined by the sensitization profile at a molecular level of plant-food allergy; some of proteins e.g. profilins are responsible for local allergic reactions, some of them e.g. lipid transfer protein - for systemic symptoms $[5,6]$.

In most cases the symptoms occur in adults with sensitization to pollen allergens being the cause of seasonal allergic rhinitis (SAR) and/or bronchial asthma, although not all patients have obvious hay fever or seasonal allergy symptoms [2, 5].

This entity can also occur in children, but there is limited information regarding the onset and sensitization patterns of pollen-food allergy and clinical manifestation of cross-reactivity in this age group. The occurrence of symptoms of pollen allergy is not as well known and ob-

Address for correspondence: Beata Cudowska MD, PhD, Department of Paediatrics, Gastroenterology, Hepatology, Nutrition and Allergology, Medical University of Bialystok, 17 Waszyngtona St, 15-274 Bialystok, Poland, phone: +48 85 745-07-10, fax: +48 85 742-38-41, e-mail: beata.cudowska@umb.edu.pl

Received: 4.01.2019, accepted: 20.07.2019. 
vious as in adults and the prevalence of allergic rhinitis and asthma is not exactly determined [4].

The relationship between exacerbation of symptoms from the respiratory tract and consumption of sensitizing foods still remains unclear and pollen-food syndrome is heterogeneous with regard to triggers, severity and response to treatment in paediatric population $[5,7,8]$. There is also a group of children sensitized to food plant derived allergens without any clinical manifestation, so the role of diet in the incidence or worsening of the symptoms is controversial [8]. On the other hand, the risk of anaphylactic episodes seems to be as important as in adults, so it is necessary to detect this type of sensitiveness early [8, 9].

\section{Aim}

The objectives of the study were to determine the prevalence of seasonal allergic rhinitis in children with pollen-related food allergy, identify foods that are frequently associated with symptoms and assess the impact of food allergens on the course of the disease and exacerbations of nasal symptoms.

\section{Material and methods}

The group of 43 children, hospitalized in our department, aged 2-14 years were enrolled in the study performed between 2016 and 2017. The inclusion criteria to the study group was IgE-mediated sensitization to pollen and homologous food allergens evaluated by measurement of specific IgE antibodies. The control group consisted of 19, age comparable, children with seasonal allergic rhinitis caused by pollen, without pollen-related food allergy.

SAR was defined as a prevalence of nasal symptoms such as: a runny or stuffy nose, sneezing, red, itchy, and watery eyes, and swelling around the eyes, triggered by pollen allergens [10, 11]. The diagnosis of SAR was based on clinical symptoms appearing seasonally according to the profile of sensitization. Patients with deformities within the face, non-allergic chronic nasopharyngeal dis-

Table 1. Population characteristics

\begin{tabular}{lccc}
\hline Characteristics & $\begin{array}{c}\text { Study } \\
\text { group } \\
n=43\end{array}$ & $\begin{array}{c}\text { Control } \\
\text { group } \\
n=19\end{array}$ & $P$-value \\
\hline Age [years], mean \pm SD & $7.54 \pm 3.38$ & $10.14 \pm 3.98$ & 0.066 \\
\hline Min.-max. & $2-14$ & $2-14$ & \\
\hline Sex, $n$ (\%): & $23(53.49)$ & $14(73.68)$ & 0.026 \\
\hline Male & $20(46.51)$ & $5(26.32)$ & 0.001 \\
\hline Female & $15(34.88)$ & $5(26.32)$ & 0.505 \\
\hline $\begin{array}{l}\text { Family history of atopic } \\
\text { disease, } n \text { (\%) }\end{array}$ & & & \\
\hline
\end{tabular}

eases, systemic and chronic diseases with non-allergic background were excluded from the study.

Some patients were tested using skin prick tests in the outpatient clinic, but to unify the inclusion criteria, all patients were tested for presence of specific IgE. Serum samples $(2 \mathrm{ml})$ were analysed for concentration of specific IgE antibodies to food and airborne allergens with a fluoroimmunoenzymatic assay (PolyCheck) according to the manufacturer's instructions. The detection limit of the system is $0.35 \mathrm{kU} / \mathrm{l}$ lgE; measurable specific IgE was defined as a positive test result if $>0.7 \mathrm{kU} / \mathrm{l}$.

Subjects were reviewed for the clinical data and history of pollen-food sensitization and SAR, skin prick test outcome, and specific IgE. Information on foods triggering clinical symptoms was acquired by the questionnaire.

The parents signed written informed consent for their children to participate in the study. The local Ethics Committee approved this study (R-I-002/160/2016).

\section{Statistical analysis}

Statistical analysis was performed by using SPSS for Windows software (version 13.1; PL). The comparison of quantitative variables was carried out using the Student's t-test in case of normally distributed data and the Mann-Whitney test in case of nonparametric data. A $p$-value $<0.05$ was considered statistically significant.

\section{Results}

\section{Patients}

Forty-three patients sensitized to pollen and homologous food allergens were qualified to the study. The population analysis regarding the age, sex and family history of allergy was presented in Table 1. The groups were comparable with respect to age, but statistically significant differences were found in terms of sex. Allergic rhinitis and asthma were the most common diseases in the families of children, according to the family history of atopy and there was no evidence of significant differences in this characteristic between study and control groups.

\section{Clinical symptoms and sensitization to airborne allergens}

Allergic rhinitis was the main symptom in (28/43) $65.12 \%$ of children with pollen-food sensitization. Tree pollen allergens were the main cause of nasal symptoms in both groups of patients and significant statistical differences were observed in the frequency of sensitization to all aeroallergens in the study group in comparison to the control one, except for sensitization to moulds (Table 2).

Analysis of the clinical status revealed that in all investigated children concomitant allergic disorders were reported: over half of them had allergic rhinitis with bronchial asthma (25/43; 58.40\%). No statistically significant 
Table 2. Comparison of sensitization to airborne allergens between study and control groups

\begin{tabular}{lccc}
\hline $\begin{array}{l}\text { Airborne } \\
\text { allergens }\end{array}$ & $\begin{array}{c}\text { Study group } \\
n(\%)\end{array}$ & $\begin{array}{c}\text { Control group } \\
n(\%)\end{array}$ & $P$-value \\
\hline Tree pollen & $40(93.02)$ & $8(42.11)$ & 0.003 \\
\hline Mites & $29(67.44)$ & $8(42.11)$ & 0.003 \\
\hline Weed pollen & $26(60.47)$ & $6(31.58)$ & 0.001 \\
\hline Cat dander & $24(55.81)$ & $4(21.05)$ & 0.0003 \\
\hline Grass pollen & $23(53.49)$ & $14(73.68)$ & 0.001 \\
\hline Dog dander & $19(44.19)$ & $4(21.05)$ & 0.001 \\
\hline Moulds & $11(25.58)$ & $2(10.53)$ & 0.480 \\
\hline
\end{tabular}

differences were noted in comparison to the control group $(58.40 \%, p=0.114)$, in contrast to the presence of atopic dermatitis ( $88.40 \%$ vs. $5.26 \%, p<0.001)$. If we analyse the frequency of asthma and atopic dermatitis in children with SAR and pollen-food sensitization, the percentages are correspondingly higher (22/28; 78.57\% and 25/28; 89.29\%).

\section{Food-related symptoms}

Plant-derived food allergens were the foods most often causing clinical symptoms in the study group (Table 3). In most cases (26/43; 60.47\%), simultaneous sensitization to pollen and animal origin allergens were confirmed. 22/43 (51.16\%) individuals were sensitized to two and more plant-derived food allergens; almost half of them were allergic to nuts; soy was the second most sensitizing food.

From the group of animal origin allergens, milk and egg white played the most important role, but among control patients sensitization to egg white was reported only in one subject (5.26\% vs. $39.53 \% ; p=0.027$ )

The clinical manifestations most often associated with sensitization to pollen-related food allergens were oral allergy syndrome (OAS) reported in 7/43 (25.58\%) subjects. In patients with pollen-food sensitization and SAR the frequency of OAS was $4 / 28(14.29 \%, p=0.237)$. In 3 children the progression towards systemic symptoms (generalized urticaria, dyspnoea) was observed after consumption of peanuts (2) and pear (1). Among the harmful allergens of OAS, apple (5 children) and nuts ( 2 children) played the main important role.

The reported association between consumption of pollen-related foods and exacerbation of atopic dermatitis was noticed in $7 / 43$ (16.28\%) children; in patients with pollen-food sensitization and SAR, the worsening of eczema after eating was observed in 5/28 (17.86\%) (the main harmful foods were nuts).

Anaphylactic reactions to food allergens were registered in (11/43) $25.58 \%$ of subjects; to cow's milk in 8 , peanuts in 2, egg white and pear in 1 patient. The mean age of children from the study group and episodes of
Table 3. Sensitization to food allergens in the study group

\begin{tabular}{lc}
\hline Food allergens & $\boldsymbol{N}(\%)$ \\
\hline Pollen-related allergens: & $43(100.0)$ \\
\hline Hazelnuts & $21(48.83)$ \\
\hline Peanuts & $19(44.19)$ \\
\hline Soy & $13(30.23)$ \\
\hline Wheat & $12(27.91)$ \\
\hline Apple & $10(23.26)$ \\
\hline Animal-related allergens: & $26(60.47)$ \\
\hline Milk & $17(65.38)$ \\
\hline Egg white & $17(65.38)$ \\
\hline Fish & $7(26.92)$ \\
\hline Meat & $5(19.23)$ \\
\hline
\end{tabular}

anaphylaxis was $8.55 \pm 3.56$ year. Anaphylactic episodes were registered only in pollen-food sensitized children.

16/43 (37.21\%) patients did not demonstrate clinical signs of food allergy during the study period (asymptomatic patients), despite sensitization to airborne and homologous food allergens; among patients from the study group who suffered from SAR this percentage was $25.0 \%$ $(p=0.010)$.

In (8/28) $25.78 \%$ of children temporal association between ingestion of pollen-related foods and nasal symptoms was observed (mainly fruits from Rosaceae family such as apple, pear, plum - in 5 cases and nuts in 3 cases); occurring also outside the pollen period. The symptoms appeared after consumption of fresh fruits; the cooked or baked form of foods did not provoke side effects.

The simultaneous sensitization to animal origin food allergens was noted in $26 / 43(60.47 \%)$ patients from the study group and in $17 / 28$ (60.71\%) of those with SAR. Only in two of them milk and egg white proteins were an additional exacerbation factor of nasal symptoms.

\section{Discussion}

The published data show that about $50-70 \%$ of patients with pollen allergy develop hypersensitivity to certain homologous food allergens in apple, celery, carrot and hazelnuts [4, 6, 7]. In children, the order of acquiring the sensitization can be reversed; usually allergy to foods appears first and then allergy to airborne allergens, consequently with clinical symptoms of pollen-food sensitization [8, 9]. Seasonal allergic rhinitis is the most common manifestation of sensitization to pollen in children and in adults, but in children with pollen-food allergy the data are still unknown $[4,8]$.

We were interested in the importance of allergy to homologous food allergens in patients with SAR and its impact on nasal symptoms. 
The data from our study have suggested that this problem could affect the youngest patients up to 3 years old; the mean age of investigated children was 7.54 years; $4(9.30 \%)$ patients were under 3 years of age and 16 (37.21\%) under 6 years of age.

The frequency of SAR in the study group has been assessed for $65.12 \%$, what confirmed that the prevalence of allergic rhinitis among children with pollen-food sensitization is high and this diagnosis is common. Tree pollen was the most frequent sensitizing allergens in both groups. In other population studies, grass was one of the main pollen allergens being the cause of SAR in children the cause of SAR $[9,10]$, but it should be remembered that the spectrum of sensitizing allergens depends on the patient's age and place of residence [11-13].

A separate problem is the clinical importance of sensitization to foods in patients sensitized to plant origin foods; especially with regard to allergic rhinitis. According to the clinical observations, it is known that some foods have an impact on the exacerbation of allergic rhinitis symptoms, but still there are controversies about the role of diet in the incidence or worsening of the symptoms [14]. In the published research carried out by Al-Rabia et al., rice, citrus fruits, black grapes and banana were identified as major allergens for inducing allergic rhinitis symptoms and the prevalence of food allergy was more than 60\% [14].

Isolated food-induced allergic rhinitis is not common as it frequently occurs together with other food allergy symptoms such as asthma or atopic dermatitis $[15,16]$. Many patients associate the ingestion of food allergens contained in dairy products, fish, meat etc. with an increased production and thickness of nasal secretion [17, 18]. Patients allergic to fruits and vegetables suffered mostly from symptoms limited to oral mucosa $[3,4,7]$.

There are limited data concerning paediatric population. The primary sensitization patterns change during life and may not correlate with clinical symptoms [19, 20]. On the one hand, the diagnosis of pollen-food sensitization is not difficult because it is usually lgE-mediated hyperreactivity and skin prick tests and assessment of specific IgE-antibodies play the most important role [4, 14]. On the other hand, the problem that still poses difficulties is proving the association between food sensitization and exacerbation of nasal symptoms. In our study, information on foods triggering nasal symptoms was acquired by the questionnaire and the data were collected during and outside the pollen period. We are aware that the results of research are limited by the subjective patients' assessment and further studies with a larger sample size and double-blind placebo controlled food challenges might be needed.

The analysis of the spectrum of sensitizing allergens showed that the investigated children were in the majority sensitized to hazelnuts, what is understandable regarding the frequency of sensitization to tree pollen. The data obtained from the questionnaire did not have the clinical relevance given the allergological tests because only 3 patients sensitized to pollen origin food allergens reported clinical symptoms after consumption of nuts.

Interestingly, the temporal association between nasal symptoms and intake of food allergens was reported only in 8 patients with pollen-related food sensitization and SAR. The limitation of obtained results can be explained by the use of treatment with antihistamines and nasal steroids during the pollen period. This may be a reason for the asymptomatic course of allergy to plant origin foods observed in one-third of patients.

Some of young patients with pollen-food allergy and SAR are also sensitized to animal origin food allergens. In the paper published by Pang et al., children with allergic rhinitis were usually allergic to milk and egg proteins [12]. In our study the subjects were sensitized to milk and egg in $65.38 \%$, but the consumption of these foods did not have implications on nasal symptoms.

Discussing the role of food allergens as a trigger factor of clinical symptoms, we should not forget about the role of component-resolved diagnostics (CRD) based on the determination of specific IgE against purified native and recombinant allergenic molecules [21]. Although this method allows to distinguish the true allergens from the cross-reactive allergen molecules and facilitate predicting the risk of systemic reactions, it is still not a routine and widely available test $[21,22]$. We are aware that lack of component-resolved diagnostics may be a limitation of our study, but it is important to note that oral food challenge and the correlation of clinical symptoms with ingested foods still have a decisive role in diagnosis of food allergy $[2,11]$. It is also worth noticing that all allergic tests can be positive to foods even if they are well tolerated and the benefits are still questionable for a number of major allergens [23].

In our study, hypersensitivity to foods in patients with SAR co-exists with OAS $[1,3,6]$. OAS is rarely seen in young children in contrast to adults; in the USA it has been estimated to be $5.0 \%$, while in some European countries it was higher than $20 \%$ [24]. It should be remembered that OAS is the local manifestation of PFS and according to other studies, the prevalence of OAS among children with sensitization to pollen was more than $5 \%$ and children were mostly sensitized to apple and nuts [24]. The results of our study confirmed these observations because the incidence of OAS, especially in patients with SAR, was relatively low (14.29\%) and SAR did not predispose to the occurrence of OAS. However, it should be noted that patients with rhinitis were more likely to have progression towards systemic symptoms.

Mastrorilli et al. divided PFS into five endotypes depending on the allergen being the primary sensitizer and showed a relationship between the severity of symptoms and PFS endotype $[9,25]$. In our study, in 3 children, the progression towards systemic symptoms was observed, however, fur- 
ther studies with a larger sample size and double-blind placebo controlled methods might be needed [26, 27].

It is difficult to identify the true prevalence of foodinduced allergic rhinitis due to one more reason: it frequently occurs in association with other food allergy symptoms such as asthma, atopic dermatitis, urticaria or gastrointestinal symptoms [15].

The major frequency of asthma among pollen allergic patients with plant food allergy has been reported not only in our investigations $(58.40 \%, p=0.114)$, but also in another study conducted by Cuesta-Herranz et al. [28].

As regards atopic dermatitis, its occurrence was statistically higher in the study group. Analysis of patient's data showed that the exacerbation of skin lesions was rarely associated with the ingestion of sensitizing foods and appeared also outside the pollen period. Comparing with other studies, this correlation is still unclear [29-31]. In our study, based on the data from the patient's history, exacerbation of skin lesions after consumption of plantderived food allergens was reported in $16.28 \%$ of children sensitized to fruit, vegetables and nuts. The frequency of atopic dermatitis in the control group was very low (5.26\%) and food allergy was not the factor that exacerbated symptoms. It should be emphasized that data from the patient's history may not be reliable in such cases and should be confirmed by the oral food challenge. On comparing these results with the others performed by Breuer et al. based on food provocation and carried out in children with sensitization to birch homologous food allergens, only one child reported the exacerbation of skin lesions after consuming sensitizing foods [29].

What is remarkable, over one-third of children were asymptomatic to plant food allergens despite pollen-food sensitization. Analysing this fact we should be aware that it indicates only the immunological response and fact of cross-sensitization, and is not the proof of clinical symptoms. Differences between clinical symptoms in children and adults with pollen-related food allergy may result from the different pattern of sensitization depending on age. It is known that adults are primarily sensitized to airborne and secondary to homologous food allergens. In young children, sometimes after the period of sensitization to food allergens, sensitization to airborne allergens appears. The time between allergy to pollen and the onset of symptoms can be very variable and range from a few days to several months or even years. It depends on the individual immunological response and the frequency of contact with harmful foods. From the phase of asymptomatic disease and good food tolerance, the patient goes into the symptomatic phase with a full manifestation of symptoms [20,32].

\section{Conclusions}

Children with seasonal allergic rhinitis and an associated plant-food allergy deserve special attention in ob- servation and management due to the high frequency of concomitant allergic diseases such as asthma and atopic dermatitis and a different pattern of IgE sensitization to several allergens. It is difficult to prove the relationship between food allergen intake and nasal symptoms as most children eliminate a priori sensitizing foods due to fear of adverse reactions. So, according to our results it should be careful in routine recommending the avoidance diet, in patients with pollen-food allergy in order to achieve better clinical outcomes.

\section{Acknowledgments}

This study was supported by grant No. N/ST/ZB/17/ 004/1143 from the Medical University of Bialystok (Poland). The study was conducted in the Department of Paediatrics, Gastroenterology, Hepatology, Nutrition and Allergology, Medical University of Bialystok, Poland.

\section{Conflict of interest}

The authors declare no conflict of interest.

\section{References}

1. Katelaris $\mathrm{CH}$. Food allergy and oral allergy or pollen-food syndrome. Curr Opin Allergy Clin Immunol 2010; 10: 246-51.

2. Von TA, Scott DR, Chin WK, et al. Differential skin test reactivity to pollens in pollen food allergy syndrome versus allergic rhinitis. Allergy Asthma Proc 2015; 36: 379-85.

3. Muluk NB, Cingi C. Oral allergy syndrome. Am J Rhinol Allergy 2018; 32: 27-30.

4. Ivković-Jureković I. Oral allergy syndrome in children. Int Den J 2015; 65: 164-8.

5. Baker MG, Sampson HA. CME Reviews: phenotypes and endotypes of food allergy: a path to better understanding the pathogenesis and prognosis of food allergy. Ann Allergy Asthma Immunol 2018; 120: 245-53.

6. Treudler R. Simon JC. Pollen-related food allergy: an update. Allergol J Intern 2017; 26: 273-82.

7. Bohle B. The impact of pollen-related food allergens on pollen allergy. Allergy 2007; 62: 3-10.

8. Ludman S, Jafari-Mamaghani M, Ebling R, et al. Pollen food syndrome amongst children with seasonal allergic rhinitis attending allergy clinic. Pediatr Allergy Immunol 2016; 27: 134-40.

9. Mastrorilli C, Tripodi C, Caffarelli S, et al. Endotypes of pollen-food syndrome in children with seasonal allergic rhinoconjunctivitis: a molecular classification. Allergy 2016; 71: 1181-91.

10. Barr JG, Al-Reefy H, Fox AT, et al. Allergic rhinitis in children. BMJ 2014; 349: g4153.

11. Hardjojo A, Shek PC, van Bever H, et al. Rhinitis in children less than 6 year of age: current knowledge and challenges. Asia Pac Allergy 2011; 1: 115-22.

12. Pang KP, Pang KA, Pang EB, et al. Food allergy and allergic rhinitis in 435 Asian patients - a descriptive review. Med J Malaysia 2017; 724: 215-20.

13. Yang LF, Cai LM, Li M, et al. A 10-year retrospective study of alterative aeroallergens sensitization spectrum in urban 
children with allergic rhinitis. Ther Clin Risk Manag 2018; 14: 409-16.

14. Al-Rabia MW. Food-induced immunoglobulin E-mediated allergic rhinitis. J Microsc Utrastruct 2016; 4: 69-75.

15. Cingi C, Demirbas D, Songu M. Allergic rhinitis caused by food allergies. Eur Arch Otorhinolaryngol 2010; 267: 1327-35.

16. Bemanian MH, Arshi S, Nabavi M. Food-induced allergic rhinitis. J Pediatr Rev 2013; 1: 61-3.

17. Malik V, Ghosh S, Woolford TJ. Rhinitis due to food allergies: fact or fiction? J Laryngol Otol 2007; 121: 526-9.

18. Seedat RY. The role of food allergy in rhinitis and nasal polyposis. Curr Allergy Clin Immunol 2015; 28: 114-7.

19. Patel D, Anvari S, Buheis M, et al. Pollen food allergy syndrome in pediatrics: a case series. Ann Allergy Asthma Immunol 2016; 117: S106.

20. Sano A, Yagami A, Inaba Y, et al. Sensitization profile of a case of pollen-food allergy syndrome. Allergol Int 2011; 60: 97-101.

21. Borres MP, Maruyama N, Sato S, et al. Recent advances in component resolved diagnosis in food allergy. Allergol Inter 2016; 65: 378-87.

22. Tuano K, Davis CM. Utility of component-resolved diagnostics in food allergy. Curr Allergy Asthma Rep 2015; 15: 32.

23. Schussler E, Kattan J. Allergen component testing in the diagnosis of food allergy. Curr Allergy Asthma Rep 2015; 15: 55.

24. Bedolla-Barajas M, Kestler-Gramajo A, Alcala-Padilla G, et al. Prevalence of oral allergy syndrome in children with allergic diseases. Allergol Immunopathol 2017; 45: 127-33.

25. Błażowski Ł, Gregorczyk-Maślanka K, Kurzawa R. Oral allergy syndrome (pollen food syndrome), anaphylaxis and molecular allergology. New endotypes and new horizons. Pol J Allergol 2016; 3: 137-43.

26. Pénard-Morand C, Annesi-Maesano I, Raherison C, et al. Prevalence of food allergy and its relationship to asthma and allergic rhinitis in schoolchildren. Allergy 2005; 60: 1165-71.

27. Jabbari Azad F, Shirkani A, Faridhosseini R. Assessment of oral allergy syndrome in patients with seasonal allergic rhinitis by food challenge test. Iran South Med J 2014; 17: 215-22.

28. Cuesta-Herranz J, Barber D, Blanco C, et al. Differences among pollen-allergic patients with and without plant food allergy. Int Arch Allergy Immunol 2010; 153: 182-92.

29. Breuer K, Wulf A, Constien A, et al. Birch pollen-related food as a provocation factor of allergic symptoms in children with atopic eczema/dermatitis syndrome. Allergy 2004; 59: 988-94.

30. Čelakovská J, Bukač J. The severity of atopic dermatitis and analysis of the food hypersensitivity reactions. Food Agricultural Immunol 2015; 26: 896-908.

31. Darsow U, Laifaoui J, Kerschenlohr K, et al. The prevalence of positive reactions in the atopy patch test with aeroallergens and food allergens in subjects with atopic eczema: a European multicenter study. Allergy 2004; 59: 1318-25.

32. Azamar-Jacome AA, Azamar-Jacome MA, Borjas-Aguilar KL, et al. Clinical-epidemiological profile of oral allergy syndrome in the population aged 6 to 18 years. Rev Alerg Mex 2017; 54: 142-52. 